\title{
Women's Empowerment and Community Norms Association on Women's Access to Health Facility Delivery in Ethiopia. A Multilevel Analysis.
}

Daniel Bekele ( $\sim$ daniadvent12@gmail.com )

Dire Dawa University

\section{Research Article}

Keywords: Health-care facility, cross-sectional survey, empowered women, health facility delivery

Posted Date: June 4th, 2021

DOl: https://doi.org/10.21203/rs.3.rs-574394/v1

License: (c) (i) This work is licensed under a Creative Commons Attribution 4.0 International License.

Read Full License 
Women's empowerment and community norms association on women's access to health facility delivery in Ethiopia. A multilevel analysis.

Daniel Bekele

Department of Statistics, College of Natural and Computational Sciences, Dire Dawa University, Dire Dawa, Ethiopia

Email: daniadvent12@gmail.com Phone: +251911518020

Corresponding author: Daniel Bekele

Email: daniadvent12@gmail.com 


\begin{abstract}
Background

Health-care facility delivery was the most critical in ensuring the provision of high-quality care and a distribution location that was ready in the case of an emergency for reproductive women. However, maternal mortality remains high in African nations, and the majority of women were still giving birth at home. This study was aimed to determine whether women's empowerment and community norms, plus other proximate factors, are related to the health facility delivery utilization of women in Ethiopia.
\end{abstract}

\title{
Methods
}

The data for this study was taken from the Performance Monitoring for Action (PMA) in Ethiopia of the 2019 cross-sectional survey. A weighted sample of 4864 women with at least one birth history, clustered within 264 clusters was used for this study. The impact of women's empowerment, cultural norms, and other proximate factors on the use of health facility deliveries among reproductive-age women across clusters in Ethiopia was studied using a two-level multilevel logistic regression.

\section{Results}

In Ethiopia, around $51 \%$ of women were delivered their most recent child at the health facility. The use of a health facility delivery was more common among empowered women and those living in where the most people encourage a health facility delivery. Older women were less likely to deliver at a health facility and women from the highest wealth quantile more likely to have a facility delivery. Those women with higher education and living in an urban area were more likely to have a health facility delivery. Women's chances of giving birth in health facilities vary significantly across the 264 clusters of Ethiopia $\left(\sigma_{u o}^{2}=2.49\right.$, p. value $\left.<0.001\right)$.

\section{Conclusions}

This study emphasizes the importance of women's empowerment and cultural norms in enhancing maternal health outcomes of women in Ethiopia. It is more important than ever that the government and development agency should invest more in women's empowerment and raising community 
consciousness about the benefits of using health facility delivery as part of a strategic intervention to improve maternal health outcomes.

\section{Background}

Health facilities deliveries were the essential ways of ensuring the availability of quality care and delivery place prepared for an emergency for reproductive women. In the middle and high-income countries, the majority of women deliver the child in health facilities. In Europe, 99 percent, Latin America, and the Caribbean 94 percent meaning over 9 in 10 births has occurred in a health facility. Despite this, in West and Central Africa and Eastern and Southern Africa still faces the huge burden of maternal and newborn deaths was highest, 54 and 60 percent of births were delivered in a health facility respectively, with 20 percent of an urban-rural gap, and 50 percent gap between women of richest and poorest wealth quantities(1).

In 2017 according to World Health Organization (WHO) report globally, 810 women are died daily due to complications of pregnancy and childbirth-related problems and 94 percent of maternal deaths occurred in the low and lower-middle-income countries(2). Similarly, in the 2017 Ethiopian Ministry of Health report, the overall maternal mortality ratio (MMR) in Ethiopia is 412 per 100,000 live births (3).

Maternal health care was provided at three stages first during their pregnancy, second during the delivery at a health facility, and third after childbirth. The second stages of maternal health care which was health facility delivery is the most important stages of ensuring maternal and child wellbeing(4)

The sustainable development goals (SDG) agenda targeted to reduce maternal mortality to 70 percent by 2030. Although effectively reducing maternal mortality in the Sub-Saharan African countries remained stalled and some of the regions show sluggish progression in reducing MMR (5). Ethiopia's Health Sector Development Plan target was to reduce MMR to 267 per 100,000 live births by the year 2015 but the country was unable to meet this target (6). It was well recognized that the huge burden of maternal mortality in Ethiopia remains the single most serious issue to the health sector. Then to succeed individual reducing and to come up with SDG target of global MMR by 2030 will require continued investment in maternal health research, programs, and policy at the global level and very focused action (7). 
In Ethiopia, as the 2019 mini EDHS report shows that the live birth occurred in a health facility were increased from 5 percent in 2005 to 48 percent in the 2019 EMDHS. Afar and Somali region have the lowest live birth occurred in health facility $28 \%$ and $23 \%$, respectively, while Addis Ababa has highest percentages of live birth occurred in a health facility was 94\%(3). These studies revealed the reason for low maternal health care service utilization was mostly associated with women's empowerment, cultural belief, and lack of awareness(8).

The study conducted in Nepal assessed the association between women's empowerment and asses to skilled birth delivery services among married women shows that media and information empowerment, economic and socio-cultural empowerment have a significant association with access to skilled birth attendants (9). Besides, the study conducted in Bangladesh using DHS data show that empowered women has more likely to believe in gender equality and refuse any violence on women's decision making such as family planning and health care services, and has a great likelihood of attending antenatal care as compared to not empowered women. Recommends women autonomy should be taken into consideration as important factors to have the highest utilization of reproductive health care services (10).

Empowered women's has made their own decision regarding healthcare and disagree with domestic violence and more likely to receive at least four antenatal care and delivery by the assistant of the skilled provider at health facilities as compared to not empowered women (11).

Women's empowerment was regarded as chiefly low in Ethiopia(12). So many studies confirmed that in Ethiopia high proportion of home delivery has happened in pastoralist and semi-pastoralist communities(13-15). For instance, the study conducted in Dubti districts of the Afar region shows that $92.6 \%$ of birth were taken place at home by traditional attendants and cultural norms, and low socioeconomic status was highly associated with the women's preference of home delivery(14).

The study conducted in rural pastoralist of the Hamar districts of southern Ethiopia revealed that; husband only decisions, women preference of traditional attendant, and not involved in the community development association were increased the risk of home delivery, further finding of the study confirmed that empowering women and promoting them to participate in community development the paths to have better institutional deliveries(15). 
Women's and Girl's empowerment (WGE) index score examined the presence of existence of choice, the exercise of choice, and achievement of choice domains across pregnancy, family planning, and sex outcomes in married women(16). However, in this study, WGEI was calculated from the decision on the existing choice of family planning(17).

Therefore, to the best of a researcher's knowledge, no prior study has been conducted that investigated the influence of women's empowerment and community norms on health facility delivery use of women of reproductive age across clusters in Ethiopia. Furthermore, this study was aiming to provide a piece of updated evidence on the status of women using health facility delivery and investigating the relationship of women's empowerment, community norms on women's preference of using a health facility delivery which was crucial outcomes for public health policymakers.

\section{Methods}

\section{Study design, area, and period}

A cross-sectional study design was used on the 2019 Performance Monitoring for Action (PMA) datasets. Ethiopia is located in Africa's Horn. It has a total area of 1,100,000 km2 and is located between $3^{\circ}$ and $14^{\circ}$ north latitude and $33^{\circ}$ and $48^{\circ}$ east longitude. There are nine regional states (Afar, Amhara, Benishangul-Gumuz, Gambela, Harari, Oromia, Somalia, Sidama, Southern Nations, Nationalities, and Peoples Region (SNNP), and Tigray) and two administrative cities (Addis Ababa and Dire Dawa). The data collection was taken place from October 2019 to December 2019.

\section{Data source and population}

The data for this study was taken from the Performance Monitoring for Action (PMA) in Ethiopia of the 2019 cross-sectional survey. PMAET was an extension of the previous PMA 2020 survey and the survey used a two-stage cluster design with urban-rural, major regions as strata. A total of 265 enumeration areas (EAs) were selected from a master sample frame of the Central Statistical Agency of Ethiopia and around 35 households old were selected randomly from within each EA. All women age 15-49 years old in a selected household were eligible for a cross-sectional survey and $8837(98.5 \%)$ of women completed the cross-sectional survey(17). For this study 4864 women 
who had at least one birth history were selected in the 2019 cross-sectional PMA Ethiopia survey, nested within 264 (EAs) enumeration areas or communities across the country.

\section{Outcome variable}

In this study, the outcome variable place of delivery of reproductive age women in age 15-49 years of their most recent childbirth, which was dichotomized into health facility birth (i.e., if the birth delivers is in any health center or hospital) or home-birth.

\section{Exposure variables}

Women's empowerment and community norms were used as the main predictors in this study. Women's empowerment index was defined in this study in terms of as the women's decision making on existence choice of family planning or their health care related to five component: 1) "If I use FP, a partner may seek another sexual partner", 2) "If I use FP, might have trouble getting pregnant when I want", 3) "If I use FP, there could be conflict in relationship or marriage", 4) "If I use FP, my children may not be born normal", 5) "If I use FP, side effects might disrupt my relationship". Adding these five variables and dividing by the number of items five gives the average score of women's empowerment index for FP, ranging from 1 to 5, was generated and categorized into three groups, women with low (1-2.8), medium (3-3.8), and high (4-5) empowerment. Community norms variables in this study were measured in terms of whether or not the community encourages women to have a health facility delivery, and which measures the likelihood of people in the community that encourage women's have a health facility delivery and the community encourages a health facility delivery (no people, most people, some people, few people). In addition to women's empowerment and community norms, several other sociodemographic variables, and some obstetric characteristics of women were also considered as confounding variables in this study. This variable includes: women's age [15-24, 25-34, 35-49], women's education [never, primary, secondary, higher], wealth quantile [lowest ,lower ,medium

,higher ,highest], birth order [1, 2-3, 4+], women's media [no, yes], women's place of residence [rural, urban] ,and women's region. 


\section{Statistical Analysis}

The 2019 cross-sectional survey of PMAET data were nested. A two-level multilevel logistic regression was employed in this study to account for the hierarchical structure of the data. Women (level 1) were nested under the clusters (EAs) (level 2) and clusters were considered as the random effects to account for unexplained variation at the community level. A binomial distribution with a logit link function was used to model the binary outcome. The data were analyzed in STATA version 16 using command melogit to estimate a parameter of interest of the four hierarchical models constructed. All analysis done in this study was weighted. A two-level multilevel logistic regression model was specified as :

$$
\log \left(\frac{\pi_{i j}}{1-\pi_{i j}}\right)=\beta_{o o}+\beta_{1} X_{1 i j}+\beta_{2} X_{2 i j}+\cdots+\beta_{p} X_{p i j}+\gamma_{1} Z_{1 j}+\gamma_{2} Z_{2 j}+\cdots+\gamma_{q} Z_{q j}+U_{o j}
$$

where: $\pi_{i j}$ is the log-odds of health facility delivery

$\beta_{o o}$ is the overall intercept

$\beta_{1} \ldots \beta_{p}$ is regression coefficient of the level one characteristics of $X_{1 i j} \ldots X_{p i j}$

$\gamma_{1} \ldots \gamma_{q}$ is regression coefficient of the level two characteristics of $Z_{1 j} \ldots Z_{q j}$

$U_{o j}$ is the community or cluster level random effect and assumed to be normally distributed with mean zero and variance equal to $\sigma_{u o}^{2}$.

The fixed effects on utilization of a health facility delivery were estimated using adjusted odds ratios from a two-level multilevel logistic regression, and random effect on utilization of a health facility delivery was estimated using the intra-class correlation coefficient (ICC) calculated as: $I C C=\frac{\sigma_{u o}^{2}}{\sigma_{u o}^{2}+\pi^{2} / 3}$ where $\sigma_{u o}^{2}$ community-level variance(18).

\section{Model building}

A total of four models were fitted in this study. The first model was fitted without any explanatory variable to examine the random variability of the clusters and to estimate the inter-class correlation coefficient (ICC). The second model was constructed to examine the effect of individual-level characteristics on the women's use of a health facility. The third model was constructed to examine 
the effect of community-level characteristics on the women's use of a health facility. The fourth model was constructed to examine effect of both individuals and community-level characteristics on the women's use of a health facility.

Finally, in this study, descriptive statistics like frequency were used. In the model building process, a univariate multilevel logistics regression was fitted for each predictor variable and the stepwise variable selection was used to prefilter variables, and those variables significant at a $20 \%$ level of significance were considered for the multivariate multilevel logistic regression model (19). Finally, in this study multivariate multilevel logistic regression model was performed to estimate the adjusted odds ratios (AORs) with $95 \%$ confidence interval (CIs) and random variation across the communities.

\section{Results}

\section{Socio-demographic characteristics of respondents}

A total of 4864 selected women (level-one) aged 15-49 years with at least one birth history nested within 264 enumeration areas (EAs) or the clusters (level-two) in Ethiopia, of these $51 \%$ of women were delivered their most recent child at health facility and $49 \%$ of women delivering at home. Almost the majority (42\%) of women were found in the age 25-34 years. The majority (52\%) of women had no formal educations and $23 \%$ of women were in the lowest wealth quantiles. In the women empowerment perspective, the majority $53 \%$ of women had a high empowerment index followed by $30 \%$ of women who had medium empowerment. The majority (75\%) of women were lived in an urban area but a majority (64\%) of them were not exposed to media. Regarding the

community norms, most people (59\%) in the community encourage health facility delivery followed by some people (19\%) in the community encourage health facility delivery. Presented in Table 1 for more details.

Table 1: Respondents weighted frequency distribution of their socio-demographic characteristics, Ethiopia, using 2019 Performance for Action (PMA) survey, 2021. 


\begin{tabular}{|c|c|c|}
\hline Characteristics & Weighted number $(\mathbf{N})$ & Weighted frequency $(\%)$ \\
\hline \multicolumn{3}{|c|}{$\overline{\text { Age of women (in a year) }}$} \\
\hline $15-24$ years & 904 & 19 \\
\hline 25-34 years & 2060 & 42 \\
\hline 35- 49 years & 1900 & 39 \\
\hline \multicolumn{3}{|c|}{ Women's educations } \\
\hline Never & 2525 & 52 \\
\hline Primary & 1604 & 33 \\
\hline Secondary & 461 & 9 \\
\hline Higher & 269 & 6 \\
\hline \multicolumn{3}{|l|}{ Wealth quantile } \\
\hline Lowest & 1114 & 23 \\
\hline Lower & 1087 & 22 \\
\hline Middle & 964 & 20 \\
\hline Higher & 896 & 18 \\
\hline Highest & 803 & 17 \\
\hline \multicolumn{3}{|c|}{ Women's and girl's empowerment } \\
\hline Low & 811 & 17 \\
\hline Medium & 1451 & 30 \\
\hline High & 2556 & 53 \\
\hline \multicolumn{3}{|l|}{ Birth order } \\
\hline 1 birth & 915 & 19 \\
\hline 2-3 birth & 1482 & 30 \\
\hline 4+ birth & 2467 & 51 \\
\hline \multicolumn{3}{|c|}{ Media exposure of women } \\
\hline No & 3099 & 64 \\
\hline Yes & 1743 & 36 \\
\hline \multicolumn{3}{|c|}{ Place of residence } \\
\hline Rural & 1202 & 25 \\
\hline Urban & 3662 & 75 \\
\hline
\end{tabular}




\section{Community encourage HFD}

No people

Most people

Some people

Few people

Women's region

Addis Ababa

Tigray

Afar

Amhara

Oromia

Somali

Benishangul- Gumuz

SNNP

Gambela

Harari

Dire Dawa

\section{Place of delivery}

Home

Health facility

\section{4}

2853

896

634

180

265

61

1124

1940

210

53

977

19

20

20

2407

2457
9

59

19

13

4

5

2

23

39

4

2

20

0.3

0.4

0.4

49

51

\section{Result of multilevel models}

Before fitting to the multivariate multilevel logistic regression models, those variables that are statistically significant at a $20 \%$ level of significance were selected using the stepwise variable selection method. Table 2 contained the results of different models considered in this study. As shown in Table 2, model 1 (model without explanatory variable) confirmed the existence of the statistically significant variation among women reproductive age across the clusters or community in the use of health facility during delivery. Furthermore, around $43.12 \%$ intra-class correlation (ICC) in the use of health facility delivery was attributable to enumeration areas (EAs) or clusters level characteristics in Ethiopia. Finally, model 4 was selected as a well-fitted model to the data 
on basis of lower AIC and log-likelihood in this study. Finally, all estimated parameter was interpreted in adjusted odds ratios (AORs).

Women with a high women's empowerment index were two times more likely to deliver at a health facility as compared to those with a low women's empowerment index (AOR=2.13, 95 \% CI: 1.65-4.50). Those women living in the community of most people who encourages a health facility delivery were almost two times more likely to have a health facility delivery as compared to those no people $(\mathrm{AOR}=2.23,95 \% \mathrm{CI}: 1.30-3.83)$.

Women aged 35 to 49 years were less likely to have a health facility deliver as compared to those aged 15-24 years old women $(\mathrm{AOR}=0.39,95 \% \mathrm{CI}$ : $0.24-0.63)$. Women from the highest wealth quantile group were more almost four times likely to have a health facility deliver as compared to those in the lowest wealth quantiles $(\mathrm{AOR}=3.80,95 \% \mathrm{CI}: 1.80-8.03)$. Women with higher educational levels were almost seven times more likely to have a health facility delivery as compared to those with no formal educations (AOR=7.39, 95\% CI: 2.88-18.94).

Table 2: Result of the multilevel logistic regression analysis of women's empowerment and community norms impact on health facility delivery, Ethiopia, 2021.

\begin{tabular}{|c|c|c|c|c|}
\hline $\begin{array}{l}\text { Characteristics } \\
\text { Fixed effects }\end{array}$ & Model 1 & $\begin{array}{l}\text { Model } 2 \\
\text { AOR }(95 \% \mathrm{CI})\end{array}$ & $\begin{array}{l}\text { Model } 3 \\
\text { AOR }(95 \% \text { CI })\end{array}$ & $\begin{array}{l}\text { Model } 4 \\
\text { AOR }(95 \% \text { CI })\end{array}$ \\
\hline \multicolumn{5}{|l|}{ Age } \\
\hline 15- $24(\mathrm{R})$ & & 1 & & 1 \\
\hline $25-34$ & & $0.87(0.60-1.25)$ & & $0.84(0.58-1.23)$ \\
\hline $35-49$ & & $0.41 * * *(0.26-0.66)$ & & $0.39 * * *(0.24-0.63)$ \\
\hline \multicolumn{5}{|l|}{ Wealth quantile } \\
\hline Lowest (R) & & 1 & & 1 \\
\hline lower & & $1.20(0.89-1.61)$ & & $1.16(0.86-1.57)$ \\
\hline Middle & & $1.54 *(1.09-2.18)$ & & $1.45 *(1.02-2.04)$ \\
\hline Higher & & $2.13^{* *}(1.28-3.55)$ & & $1.85 *(1.10-3.11)$ \\
\hline Highest & & $6.37 * * *(3.07-13.20)$ & & $3.80 * * *(1.80-8.03)$ \\
\hline \multicolumn{5}{|c|}{ Women's Empowerment } \\
\hline Low (R) & & 1 & & 1 \\
\hline
\end{tabular}


Medium

High

Women's educational level

Never (R)

Primary

Secondary

Higher

Birth order

$1(\mathrm{R})$

2-3

$4+$

Women's region

Addis Ababa (R)

Tigray

Afar

Amhara

Oromia

Somali

Benishangul- Gumuz

SNNP

Gambela

Harari

Dire Dawa

Residence

Rural (R)

Urban

Community encourage HFD

No people (R)

Most people

Some people

Few people
0.85 (0.62-1.16)

$3.13(1.86-5.49)$

$0.87 *(0.65-0.97)$

$2.13 * *(1.65-4.50)$

1

$1.38 *(1.04-1.85)$

$2.40 * *(1.35-4.26)$

$7.87 * * *(3.03-20.44)$

1

$1.38 *(1.03-1.85)$

$2.26 * *(1.27-4.02)$

$7.39 * * *(2.88-18.94)$

1

$0.64 *(0.45-0.91)$

$0.53 * * *(0.37-0.75)$

1

0.65(0.23-1.87)

$0.05 * *(0.01-0.17)$

$0.23 * *(0.08-0.64)$

$0.15 * * *(0.06-0.42)$

$0.06 *(0.01-0.55)$

$0.27 *(0.08-0.90)$

$0.23 * *(0.08-0.66)$

0.37(0.10-1.02)

0.42(0.11-1.62)

$0.65(0.23-1.81)$
1

$0.61 * *(0.43-0.89)$

$0.52 * * *(0.36-0.75)$

1

1.21(0.44-3.31)

$0.14 * * *(0.05-0.44)$

0.42(0.16-1.09)

$0.30 *(0.12-0.76)$

$0.13 *(0.02-0.89)$

0.40(0.13-1.29)

0.45(0.17-1.19)

0.51(0.14-1.80)

0.71(0.20-2.50)

0.92(0.35-2.45)

$\begin{array}{ll}1 & 1 \\ 11.68 * * *(7.23-18.86) & 3.15 * * *(1.73-5.74)\end{array}$

1

$2.10 * *(1.23-3.56) \quad 2.23 * *(1.30-3.83)$

$1.82 *(1.12-2.93) \quad 1.83 * *(1.16-2.89)$

$1.81 *(1.13-2.91) \quad 1.76 *(1.08-2.86)$ 


\begin{tabular}{lllll}
\hline Random effects & Model 1 & Model 2 & Model 3 & Model 4 \\
\hline Variance (SE) & $2.49^{* * *}(0.39)$ & $1.38^{* * *}(0.25)$ & $1.34^{* * *}(0.22)$ & $1.16^{* * *}(0.22)$ \\
ICC $(\%)$ & 43.12 & 29.47 & 29.00 & 26.12 \\
PCV $(\%)$ & Reference & 44.58 & 46.18 & 53.41 \\
\hline Model fitness & Model 1 & Model 2 & Model 3 & Model 4 \\
\hline Log-likelihood & -4176.39 & -3762.38 & -4084.61 & -3714.46 \\
AIC & 8356.80 & 7574.80 & 8181.20 & 7486.90 \\
\hline
\end{tabular}

SE: Standard errors in parentheses ICC: Intra-class correlation HFD: health facility delivery

$* \mathrm{p}<0.05, * * \mathrm{p}<0.01, * * * \mathrm{p}<0.001 \quad$ AOR: Adjusted odds ratio $\mathrm{R}:$ Reference category

Those women having more than four birth orders were less likely to deliver in a health facility as compared to those with one birth order ( $\mathrm{AOR}=0.52,95 \%$ CI: 0.36-0.75). Women living in Somali region, Afar region, and Oromia region were $87 \%, 86 \%, 70 \%$ less likely to have a health facility delivery as compared to those in Addis Ababa (AOR=0.13,95\% CI: 0.02-0.89), (AOR=0.14, 95\% CI: $0.05-0.44),(\mathrm{AOR}=0.30,95 \% \mathrm{CI}$ : 0.12-0.76) respectively. Those women living in an urban area were almost three times more likely to have a health facility delivery as compared to those in the rural(AOR=3.15, $95 \%$ CI: 1.73-5.74).

\section{Discussion}

Women's empowerment and community norms were found to be significantly associated with the use of a health facility during delivery in this study. The findings from this study indicated that a variation in the use of health facility delivery among women across the clusters of Ethiopia. In adjusted multilevel logistics regression, those women with a high empowerment index and living in the community where the most people encourage a health facility delivery were more likely to use a health facility during their delivery, these findings were consistent with other studies $(20,21)$. In Ethiopia, community norms take a loan share over individual preference and this a reason behind women's living in the community where the most people encourage having a health facility deliver were more likely to use a health facility to deliver during their childbirth, which agrees with the findings of the study(22). 
Those women with higher educational levels and the highest wealth quantile category were found to be more likely to use a health facility delivery, which was consistent with the findings of the study(23). Women's with higher education level have higher odds of using health facility during their childbirth and they are more empowered in making the decision about their healthcare than any else (23).

The findings of this study also show that women in the higher age category, more than four birth orders were found to be fewer odds of using a health facility delivery. This finding was consistent with other studies $(8,24)$. Women living in the Afar region, Somali region, and Oromia region are less odd of using a health facility during their childbirth as compared to those living in Addis Ababa, which needs health policy improvements in terms of empowering women and improving community norms towards seeking health services.

\section{Strengths and Limitations}

As result, this study was disseminated from the 2019 cross-sectional survey of performance monitoring for action Ethiopia datasets, which makes more reliable the generalizability of findings to women of reproductive age groups across the clusters of Ethiopia. However, this study has a limitation that unable to make any causal relationships between variables due to the cross-sectional nature of the data. The PMA data was also collected by asking women to recall their most recent status of health services utilization, therefore recall bias was expected in the data.

\section{Conclusion}

The study findings depicted that various factors that contributed to the use of health facility deliveries. The findings of this study unfold that empowered women and those living in where more people in the community encourage a health facility delivery were high likelihood of using health facility delivery in addition to other proximate factors: women with highest educational level, living in an urban and highest wealth quantiles. Women in the older age group have more than four birth orders and living in the Afar, Somali, Oromia region were negatively associated with the use of health facilities at their most childbirth. Empowering women's ability to make decisions in their health care and emphasizing the value of delivering at a health facility was the best way to improve the individual and community use of a health facility. This study further recommends health-related initiatives that addressed gaps in women's empowerment, schooling, 
rural-urban discrepancies, and the socioeconomic background policies that would benefit the whole communities as well as that of individuals.

\section{Declarations}

\section{Ethics approval and consent to participate}

Performance Monitoring for Action (PMA) Ethiopia was received ethical approval from the Addis Ababa University School of Public Health Institutional Review Board (IRB). The requested data were treated strictly confidential and were used only for this study.

\section{Consent for publication}

'Not applicable'

\section{Availability of data and materials}

All datasets used in this study was available at (https://www.pmadata.org/countries/ethiopia ) PMA website.

\section{Competing interests}

The authors declared that they have no competing interests.

\section{Funding}

The study was funded by the Performance for Action (PMA) Ethiopia project. The funding body has no role in data extraction, statistical analysis, and interpretation of the data or writing of this manuscript.

\section{Acknowledgments}

I would like to thank Performance for Action (PMA) for providing this data.

\section{Reference}


1. UNICEF. Delivery care [Internet]. Delivery Care. 2018 [cited 2021 Mar 28]. Available from: https://data.unicef.org/topic/maternal-health/delivery-care/\#status

2. WHO, UNICEF, UNFPA WBG and the UNPD. WHO | Maternal mortality: Levels and trends [Internet]. Who. 2019 [cited 2021 Mar 28]. p. 104. Available from: https://www.who.int/reproductivehealth/publications/maternal-mortality-2000-2017/en/

3. Ethiopian Public Health Institute (EPHI) [Ethiopia] and ICF. Ethiopia Mini Demographic and Health Survey 2019: Key Indicators. Journal of Chemical Information and Modeling. 2019;53(9):1689-99.

4. World Health Organization. maternal health servie. 2020;8.

5. WHO. Strategies toward ending preventable maternal mortality (EPMM). Geneva: World Health Organization, 2015 [Internet]. 2016 [cited 2021 Mar 28]. Available from: https://www.paho.org/en/documents/strategies-toward-ending-preventable-maternalmortality-epmm

6. FMOH. Health Sector Development Program IV. Annual performance report. Addis Ababa [Internet]. 2010 [cited 2021 Mar 28]. Available from: https://www.scirp.org/(S(i43dyn45teexjx455qlt3d2q))/reference/ReferencesPapers.aspx?R eferenceID $=1800411$

7. Tesfaye S. A national scoping exercise and strategic recommendations for working with individuals, families and communities to improve maternal and neonatal health in Ethiopia [Internet]. Vol. 24, Ethiopian Journal of Health Development. 2010 [cited 2021 Mar 28]. p. 89-91. Available from: https://www.africabib.org/rec.php?RID=Q00050310

8. Solomon S, Mark S, Merijn G, Yilma M, Michael T. Why do women prefer home births in Ethiopia? BMC pregnancy and childbirth [Internet]. 2013;13:5. Available from: http://ovidsp.ovid.com/ovidweb.cgi?T=JS\&PAGE=reference \&D=medl\&NEWS=N\&AN= 23324550

9. Khatiwada J, Muzembo BA, Wada K, Ikeda S. Dimensions of women's empowerment on access to skilled delivery services in Nepal. BMC Pregnancy and Childbirth [Internet]. 
2020 Dec 1 [cited 2021 Mar 28];20(1). Available from:

https://pubmed.ncbi.nlm.nih.gov/33059624/

10. Haque SE, Rahman M, Mostofa MG, Zahan MS. Reproductive Health Care Utilization among Young Mothers in Bangladesh: Does Autonomy Matter? Women's Health Issues [Internet]. 2012 Mar [cited 2021 Mar 28];22(2). Available from:

https://pubmed.ncbi.nlm.nih.gov/21968029/

11. Osorio AM, Bolancé C, Madise N. Community socioeconomic context and its influence on intermediary determinants of child health: Evidence from Colombia. Journal of Biosocial Science [Internet]. 2015 Jan 13 [cited 2021 Mar 29];47(1):1-27. Available from: https://pubmed.ncbi.nlm.nih.gov/24555557/

12. Mekonnen AG, Odo DB, Nigatu D, Sav A, Abagero KK. Women's empowerment and child growth faltering in Ethiopia: evidence from the Demographic and Health Survey. BMC Women's Health [Internet]. 2021;21(1):1-9. Available from: https://doi.org/10.1186/s12905-021-01183-X

13. Wako WG, Kassa DH. Institutional delivery service utilization and associated factors among women of reproductive age in the mobile pastoral community of the Liban District in Guji Zone, Oromia, Southern Ethiopia: A cross sectional study. BMC Pregnancy and Childbirth [Internet]. 2017 May 15 [cited 2021 Mar 29];17(1):144. Available from: http://bmcpregnancychildbirth.biomedcentral.com/articles/10.1186/s12884-017-1325-5

14. Biza N, Mohammed H. Pastoralism and antenatal care service utilization in Dubti District, Afar, Ethiopia, 2015: A cross-sectional study. Pastoralism [Internet]. 2016 Dec 1 [cited 2021 Mar 29];6(1):15. Available from: http://pastoralismjournal.springeropen.com/articles/10.1186/s13570-016-0062-0

15. Wondimu MS, Woldesemayat EM. Determinants of home delivery among women in rural pastoralist community of hamar district, southern ethiopia: A case-control study. Risk Management and Healthcare Policy [Internet]. 2020 [cited 2021 Mar 29];13:2159-67. Available from: /pmc/articles/PMC7575064/

16. Prata N, Fraser A, Huchko MJ, Gipson JD, Withers M, Lewis S, et al. Women's 
empowerment and family planning: A review of the literature. Journal of Biosocial Science. 2017;49(6):713-43.

17. Shiferaw S, Seme A, Zimmerman L. Performance Monitoring for Action. 2020; Available from: https://www.pmadata.org/about

18. Hox JJ. 'Multilevel Analysis : Techniques and Applications', in Quantitative methodology series. Third edition [Internet]. 2010 [cited 2021 Apr 9]. Available from:

https://www.google.com/search?q=Hox\%2C+J.+J.+(2010b)+'Multilevel+Analysis+\%3A +Techniques+and+Applications'\%2C+in+Quantitative+methodology + series. + Third + edit $\& \mathrm{rlz}=1 \mathrm{C} 1 \mathrm{AVFC}$ _enET890ET923\&oq $=\mathrm{Hox} \% 2 \mathrm{C}+\mathrm{J} .+\mathrm{J} .+(2010 \mathrm{~b})+{ }^{\circ}$ Multilevel + Analysis \% $3 \mathrm{~A}+$ Techniques+and+Applicati

19. Heinze G, Wallisch C, Dunkler D. Variable selection - A review and recommendations for the practicing statistician [Internet]. Vol. 60, Biometrical Journal. Wiley-VCH Verlag; 2018 [cited 2021 Apr 9]. p. 431-49. Available from: http://doi.wiley.com/10.1002/bimj.201700067

20. Adhikari R. Effect of Women's autonomy on maternal health service utilization in Nepal: A cross sectional study. BMC Women's Health. 2016;16(1):1-8.

21. Nwogwugwu N. Women's Empowerment and Women's Health in Africa. In: The Palgrave Handbook of African Women's Studies [Internet]. Springer International Publishing; 2020 [cited 2021 Apr 10]. p. 1-19. Available from: https://doi.org/10.1007/978-3-319-77030-7_160-1

22. Mochache V, Wanje G, Nyagah L, Lakhani A, El-Busaidy H, Temmerman M, et al. Religious, socio-cultural norms and gender stereotypes influence uptake and utilization of maternal health services among the Digo community in Kwale, Kenya: A qualitative study. Reproductive Health [Internet]. 2020 May 24 [cited 2021 Apr 10];17(1):71. Available from: https://reproductive-healthjournal.biomedcentral.com/articles/10.1186/s12978-020-00919-6

23. Tiruneh FN, Chuang KY, Chuang YC. Women's autonomy and maternal healthcare service utilization in Ethiopia. BMC Health Services Research. 2017 Nov 13;17(1). 
24. Tiruneh FN, Chuang KY, Chuang YC. Women's autonomy and maternal healthcare service utilization in Ethiopia. BMC Health Services Research [Internet]. 2017 Nov 13 [cited 2021 Apr 10];17(1):718. Available from:

https://bmchealthservres.biomedcentral.com/articles/10.1186/s12913-017-2670-9 